\title{
Pairing in population imbalanced Fermion systems
}

\author{
M. J. Wolak ${ }^{1}$, V. G. Rousseau ${ }^{2}$, G. G. Batrouni ${ }^{1,3}$ \\ ${ }^{1}$ Centre for Quantum Technologies, National University of Singapore; 2 Science Drive 3 Singapore 117542 \\ ${ }^{3}$ Department of Physics and Astronomy, Louisiana State University, Baton Rouge, Louisiana 70803, USA and \\ ${ }^{3}$ INLN, Université de Nice-Sophia Antipolis, CNRS; 1361 route des Lucioles, 06560 Valbonne, France
}

\begin{abstract}
We use Quantum Monte Carlo (QMC) simulations to study the pairing mechanism in a onedimensional fermionic system governed by the Hubbard model with attractive contact interaction and with imbalance between the two spin populations. This is done for the uniform system and also for the system confined in a harmonic trap to compare with experiments on confined ultracold atoms. In the uniform case we determine the phase diagram in the polarization-temperature plane and find that the "Fulde-Ferrell-Larkin-Ovchinnikov" (FFLO) phase is robust and persists to higher temperature for higher polarization. In the confined case, we also find that the FFLO phase is stabilized by higher polarization and that it is within the range of detection of experiments currently underway.
\end{abstract}

The best understood mechanism for pair formation of fermions is the BCS mechanism ${ }^{1}$ where two fermions with opposite spin and equal but opposite momenta form a pair with zero center-of-mass momentum. Shortly after the development of the BCS theory of superconductivity, the question of pair formation in polarized superconducting systems, i.e. where the populations of the two spin states are imbalanced, was addressed independently by Fulde and Ferrel$^{2}$ (FF), Larkin and Ovchinnikov $\underline{3}$ (LO) and Sarma ${ }^{4}$. Initially, the question was motivated by interest in the nature of superconductivity in the presence of a magnetic field but since then other instances where such a mechanism intervenes have become of interest. For example, in the interior of supermassive neutron stars, quarks of various colors may form pairs which are not colorless thus leading to what is known as "color superluid" $\underline{\underline{5}}$. Another situation of major current experimental interest is in systems of confined ultra-cold fermionic atoms such as ${ }^{6} \mathrm{Li}$ or ${ }^{40} \mathrm{~K}$. Such experiments have now reported the presence of pairing in the case of unequal populations 6.7 in three-dimensional cigar shaped traps and in one dimensional traps 8 . However, the precise nature of the pairing has not yet been elucidated experimentally.

On the theoretical side, many methods have been used ranging from mean field $\underline{9}$ to effective Lagrangian ${ }^{10}$ to Bethe ansatz ${ }^{11}$. The two competing mechanisms for pair formation in the population imbalanced case are the FFLO and the Sarma mechanisms. In the former, the bosonic pairs form with non-zero center of mass momentum equal to the difference in the Fermi momenta, $\left|k_{F 1}-k_{F 2}\right|$, of the two populations. This leads to the formation of a standing wave in the order parameter whose wave vector is $\left|k_{F 1}-k_{F 2}\right|$. With the Sarma mechanism, majority fermions whose momenta equal the Fermi momentum of the minority, are promoted to higher momentum levels thus forming a breach in the Fermi distribution of the majority population. This breach allows majority fermions with momentum equal to the Fermi momentum of the minority to pair up with minority fermions forming pairs with zero center of mass momentum. Extensive numerical work on the one dimensional system us- ing Quantum Monte Carlo (QMC) ${ }^{12,13}$, and the Density Matrix Renormalization Group (DMRG) 14 ) has demonstrated that, in the ground state, population imbalance leads to a robust FFLO phase over a very wide range of polarization and interaction strengths. The Sarma phase was not detected in these numerical works.

The stability of the FFLO phase at finite temperatures has been adressed with mean field calculations ${ }^{15}$ which can be unreliable in low dimension where quantum fluctuations are large. Recently we addressed this question ${ }^{16}$ using exact quantum Monte Carlo (QMC) simulation which is the focus of this presentation.

In order to study the pairing mechanism of fermions in an optical lattice, we consider the one-dimensional fermionic Hubbard Hamiltonian,

$$
\begin{aligned}
H= & -t \sum_{i \sigma}\left(c_{i \sigma}^{\dagger} c_{i+1 \sigma}+c_{i+1 \sigma}^{\dagger} c_{i \sigma}\right)-\sum_{i}\left(\mu_{1} \hat{n}_{i 1}+\mu_{2} \hat{n}_{i 2}\right) \\
& +U \sum_{i}\left(\hat{n}_{i 1}-\frac{1}{2}\right)\left(\hat{n}_{i 2}-\frac{1}{2}\right) \\
& +V_{T} \sum_{i}\left(x_{i}-\frac{L}{2}\right)^{2}\left(\hat{n}_{i 1}+\hat{n}_{i 2}\right)
\end{aligned}
$$

where $c_{i \sigma}^{\dagger}$ and $c_{i \sigma}$ are fermion creation and annihilation operators on lattice site $i$ satisfying the usual anticommutation relation, $\left\{c_{i \sigma}, c_{j \sigma^{\prime}}^{\dagger}\right\}=\delta_{i, j} \delta_{\sigma, \sigma^{\prime}}$. The fermionic species are labeled by $\sigma=1,2$ and $\hat{n}_{i \sigma}=c_{i \sigma}^{\dagger} c_{i \sigma}$ is the corresponding number operator. The energy scale is set by taking the hopping parameter $t=1$. The contact interaction strength $U$ is negative since we are interested in pair formation in the attractive model. The last term describes the confining harmonic trap which is centered at the midpoint, $L / 2$, of the $L$-site lattice. We take periodic boundary conditions. Our QMC results were obtained using the Determinant QMC algorithm 17 (DQMC) and the Stochastic Green Function (SGF) technique ${ }^{18}$. Specifically, we used the DQMC algorithm to determine the phase diagram in the polarizationtemperature $(P, T)$ plane because of its good convergence properties for large systems. This algorithm functions 
in the grand canonical ensemble where the chemical potential for each species is tuned to obtain the desired polarization. When the populations are imbalanced, this algorithm suffers from the sign problem even with the attractive interaction, as is the case here. However, in our simulations, the average sign never went below around 0.3 at the highest polarizations and lowest temperatures. This allowed us to study the system under rather extreme conditions. The SGF algorithm was used for all other simulations including all simulations of the confined system because this algorithm functions in the canonical ensemble, where the populations are fixed, which corresponds to the experimental situation. In addition, we have verified 19 that for the system sizes and fillings we simulated, the grand canonical (DQMC) and canonical (SGF) ensembles give the same results. Typical simulations, with DQMC or SGF, took from five to seven days each on a $3 \mathrm{GHz}$ processor. The simulations were performed locally on our cluster with 102 cores.

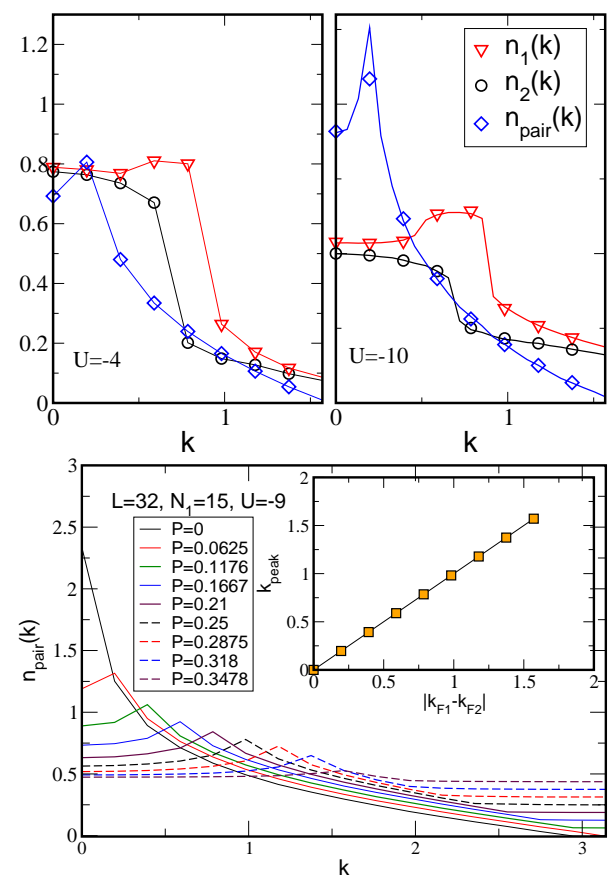

FIG. 1: Top: The momentum distributions for the majority, minority and pair populations in a system of $L=32$ sites, majority population $N_{1}=9$, minority $N_{2}=7, \beta=64$. The pair momentum distribution exhibits a distinct maximum at nonzero momentum. Bottom: The pair momentum distribution for several polarizations $P$. The inset shows that the position of the FFLO peak is equal to $\left|k_{\mathrm{F} 1}-k_{\mathrm{F} 2}\right|$.

Using these algorithms, we calculate the real space Green functions of each species, $G_{\sigma}$, and the pair green function, $G_{\text {pair }}$,

$$
\begin{aligned}
G_{\sigma}(l) & =\left\langle c_{j+l \sigma}^{\dagger} c_{j \sigma}\right\rangle, \\
G_{\text {pair }}(l) & =\left\langle\Delta_{j+l}^{\dagger} \Delta_{j}\right\rangle, \\
\Delta_{j} & =c_{j 2} c_{j 1},
\end{aligned}
$$

where $\Delta_{j}$ destroys a pair on site $j$. The Fourier transform of $G_{\sigma}(l)$ yields the momentum distributions $n_{\sigma}(k)$ and the transform of $G_{\text {pair }}(l)$ leads to the pair momentum distribution, $n_{\text {pair }}(k)$, a central quantity in this work. In the non-interacting limit, the Fermi momentum of a population is given by $k_{F \sigma}=\frac{N_{\sigma}-1}{2} \frac{2 \pi}{L}$, where $L$ is the number of sites and $N_{\sigma}$ the number of particles. The Fermi energy is given in the uniform case by $\epsilon_{F}=t k_{F}^{2}=$ $T_{F}$ where $T_{F}$ is the Fermi temperature. The polarization is defined as $P=\left(N_{1}-N_{2}\right) / N$ where $N=N_{1}+N_{2}$ is the total number of particles.

In Fig 1 we show results for the uniform system $\frac{12}{2}$, $V_{T}=0$. The top panels show the momentum distributions for the majority and minority populations and for the pairs at $U=-4 t,-10 t$. We see that the Fermi "surfaces" for the two populations are sharply defined and that the peak in the pair momentum distributions is at nonzero momentum, $k_{\text {peak }}$ : This is a clear signal for FFLO pairing. It is interesting to note the deformation of the momentum distribution of the majority population: A bump develops in $n_{1}(k)$ for $k>k_{F 2}$. As the Fermi distributions of the minority and majority start to match up more closely as $U$ becomes more attractive, the excess unpaired majority particles populate the states with momenta larger than the minority Fermi momentum. This give the bump in $n_{1}\left(k>K_{F 2}\right)$. The lower panel of Fig 1 shows $n_{\text {pair }}(k)$ for several polarizations. It is clearly seen that as $P$ increases so does $k_{\text {peak }}$, the momentum at which $n_{\text {pair }}(k)$ peaks. In the inset we display $k_{\text {peak }}$ versus $\left|k_{\mathrm{F} 1}-k_{\mathrm{F} 2}\right|$ which shows that in fact $k_{\text {peak }}=\left|k_{\mathrm{F} 1}-k_{\mathrm{F} 2}\right|$ as predicted by the FFLO scenario. The nonzero value of the pair center of mass momentum means that its Fourier transform $G_{\text {pair }}(l)$ (Eq. 3D) oscillates 12 with wavelength $\lambda=2 \pi /\left|k_{\text {peak }}\right|$. This means that in the FFLO phase the system is not homogeneous, it consists of pair-rich regions and regions depleted in pairs but rich in the excess unpaired majority population.

Such calculations were done for several values of $U$ using DMRG 14 and $\mathrm{QMC}^{12}$ and lead to the conclusion that, in the ground state, the polarized system is always in the FFLO phase and the Sarma mechanism does not intervene. The question then arises as to the effect of finite temperature: Will FFLO survive at $T \neq 0$ and how robust is this phase?

To this end, we studied, using QMC simulations, what happens to the FFLO peak as the temperature is increased. The inset in Fig. 2 shows this peak for the case $N_{1}=13$ and $N_{2}=7(P=0.3)$. We see that as $T$ increases, the FFLO peak gets lower and eventually disappears at $\beta=1 / T \approx 5$ which signals the destruction of the FFLO phase by thermal fluctuations. But how is FFLO destroyed? The two possibilities are (a) the pairs are broken or (b) the pairs are still formed but thermal fluctuations make the system homogeneous resulting in a peak at $k=0$ for $n_{\text {peak }}(k)$. To destroy the pairs, the thermal energy should be of the order of the pair binding energy, i.e. $T \sim|U|$. However, we see from Fig. 2 that FFLO 


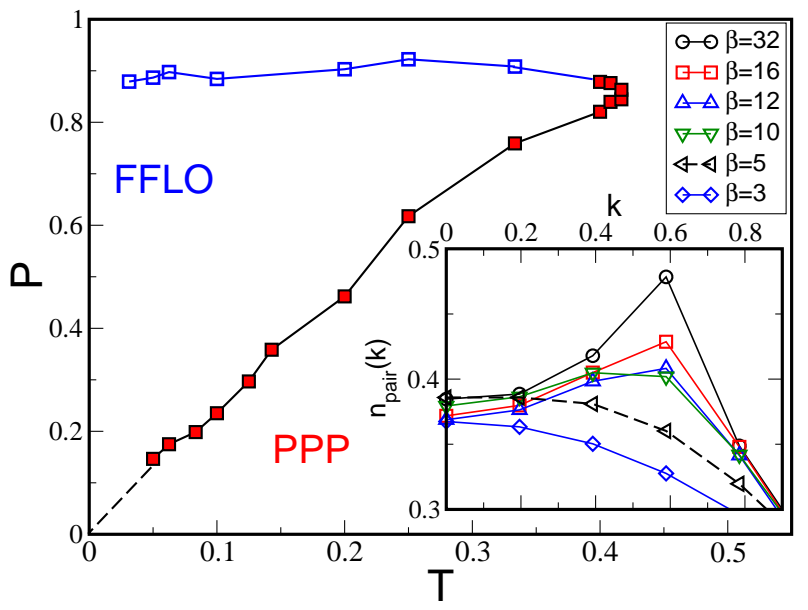

FIG. 2: The phase diagram at quarter filling, $N=L / 2$, with $U=-3.5 t$ and $L=50$. The Fermi temperature at this filling is $T_{\mathrm{F}}=0.5 t$. We see that the FFLO phase is robust and persists over a wide range of $P$ and $T$. Increasing $P$ stabilizes FFLO up to higher T. PPP stands for Polarized Paired Phase, see text for a discussion. The inset shows the behavior of the FFLO peak as $T$ is increased for the case $N_{1}=13, N_{2}=7$, i.e. $P=0.3$. We see that the FFLO peak goes down as $T$ increases and vanishes at $\beta=1 / T \approx 5$.

is always destroyed at $T \ll|U|$. We conclude, therefore, that thermal fluctuations destroy FFLO by making the system homogeneous not by destroying the pairs; we denote this phase by Polarized Paired Phase (PPP). In this way, we determine the phase diagram of the system, shown in Fig_ at quarter filling $N=N_{1}+N_{2}=L / 2$. The figure shows clearly that the FFLO phase is very robust extending over a wide range of $P$ and $T$. Increasing $P$ stabilizes FFLO up to higher $T$ while at low $P$ even a small increase in $T$ destroys it. Consequently, for small $P$ one needs to simulate the system at exceedingly low $T$ to see FFLO. This increases the simulation time and sets a limit on the lowest practical $P$. The dotted line in Fig. 2] schematizes the phase boundary at very low $P$. At the other extreme, $P \rightarrow 1$, the system is primarily made of one population with very few minority particles. This makes the FFLO signal very difficult to see. The open symbols in Fig. 2 denote the highest $P$ we were able to examine, the system up to those values is still FFLO.

Continuing earlier work in higher dimension ${ }^{6.7}$, the Rice group $\underline{\underline{8}}$ recently reported on experiments in arrays of one dimensional tubes of confined Fermionic atoms $\left({ }^{6} \mathrm{Li}\right)$ with imbalanced populations. Along the tube, the atoms were confined with a trap frequency $\omega_{z}=2 \pi \times 200 \mathrm{~Hz}$; in the central tube, the total number of atoms at zero polarization was approximately 250 and the temperature was estimated at $T / T_{F} \approx 0.1$ where the Fermi temperature $T_{F}$ is obtained from the Fermi energy $\epsilon_{F}=$ $(1 / 2+\hbar \omega) N / 2$. The pair binding energy, $\epsilon=\hbar^{2} / m a_{1 D}$ (where $a_{1 D}$ is the effective one-dimensional scattering length), was estimated to be $\epsilon / \epsilon_{F} \approx 5.3$.

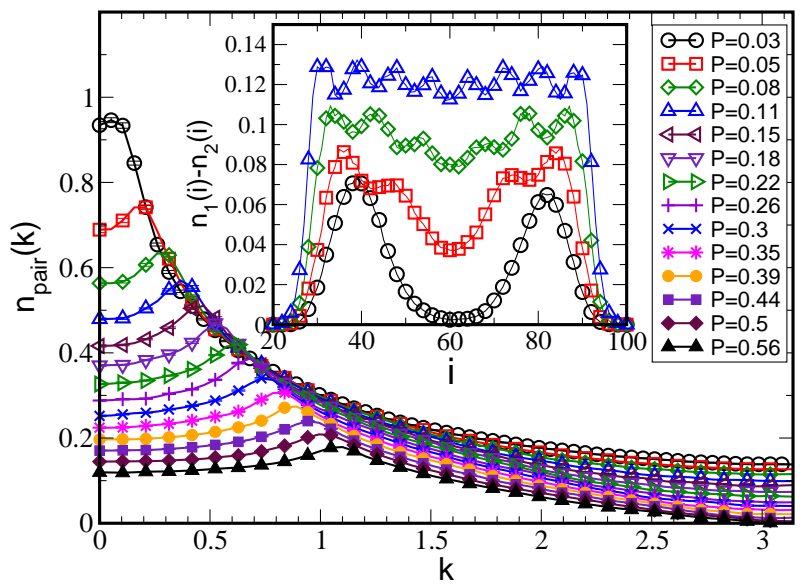

FIG. 3: The pair momentum, $n_{\text {pair }}(k)$, versus $k$ for several polarizations of the confined system, $V_{T}=0.0007 t, L=120$, $U=-10 t$ and $\beta=64$. The majority population is $N_{1}=39$ and the minority, $N_{2}$, is varied to tune $P$. Inset: Profile of the populations difference for the smallest four polarizations. See the text for a discussion of the oscillations.

We now present QMC results for the fermionic Hubbard model, Eq. (11), in the presence of the confining trap. To make contact with the experiment we introduce the trapping potential in Eq. (11) $V_{T}=0.0007 t$ which corresponds to $\hbar \omega_{z}=2 \sqrt{t V_{T}}$. The total number of particles in our simulations for balanced populations is 78 , to be compared with 250 in the experiment. We performed our simulations in the temperature range $0.008 \leq T / T_{F} \leq 0.25$ which includes the temperature at which the experiments were performed, $T / T_{F}=0.1$. In addition, to place our system in the same coupling parameter regime as the experiments, we present our results for a coupling strength of $U=-10 t . \quad U$ is the "pair binding energy" and the value we have chosen gives $|U| / \epsilon_{F}=4.8$, close to the experimental value.

In Fig 3 we show, as we did in the uniform case Fig 1 , the pair momentum distribution, $n_{\text {pair }}(k)$ for several $P$ values. We see that $k_{\text {peak }} \neq 0$ and that its value increases with $P$ as predicted by the FFLO picture. These results were obtained at $\beta=64$ and essentially represent the ground state behavior of the system. Therefore, the presence of the trap does not change the nature of the phase in the ground state, it remains FFLO when the system is polarized and is robust. The inset in Fig. 3 shows the difference between the majority and minority density profiles for the four lowest $P$ values we examined. We see that the difference is oscillatory; the wavelength of the oscillations is in fact given by $\lambda=2 \pi /\left|k_{\mathrm{F} 1}-k_{\mathrm{F} 2 \mid}\right|$. For example, we see that for the smallest $P$ we show, one wavelength fits in the system and for the $P=0.11$ case four wavelengths fit. This is a nice visual confirmation that the FFLO state is not homogeneous.

The effect of finite temperature is examined, as before, by studying the behavior of the FFLO peak as $\beta=1 / T$ is decreased (increased). We show in Fig 4 two $P$ cases. 

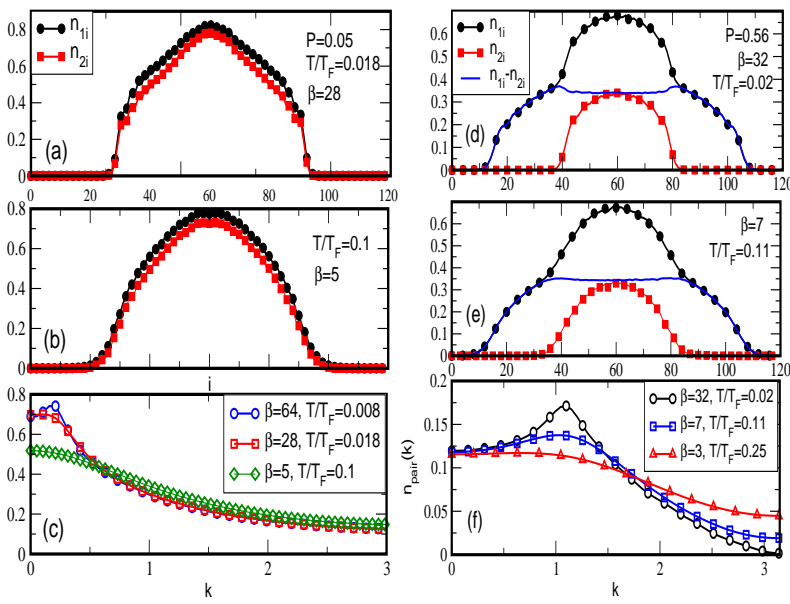

FIG. 4: Density profiles for $P=0.05$ at $T / T_{F}=0.01$ (a) and $T / T_{F}=0.1$ (b). The pair momentum distribution for three $T$ values (c) shows that the FFLO peaks disappears for $T / T_{F}=0.018$ which is much lower than what is feasible experimentally. The high polarization case, $P=0.56$ is shown in (d) and (e) for $T / T_{F}=0.02$ and 0.11 . The pair momentum distribution (f) shows that for $P=0.56$ the FFLO peak survives even for $T / T_{F}>0.1$, the experimental value.

On the left, (a) and (b) show how the density profiles, i.e. the local density in the trap, for $P=0.05$ change as the system is heated. We see that the profiles get more rounded as $T$ increases. The pair momentum distribution (c) shows that the FFLO peak disappears by the time $T=0.018 T_{F}$. This temperature is very low and is not accessible experimentally. However, the high polarization case, $P=0.56$, shown in (d) and (e) behaves differently. Here too, the profiles get rounded as $T$ increases, but we see in (f) that the FFLO peak survives for $T>0.1 T_{F}$, the experimental value. This means that with presently attainable experimental temperatures, the FFLO phase may be observed. This increased stability of FFLO with increased $P$ is consistent with the phase diagram we found for the uniform case, Fig 2 .

In this paper we have examined the pairing mechanism in fermionic systems with imbalanced populations both in the absence and presence of a confining trap which breaks translational invariance. We showed that the dominant pairing mechanism in the ground state and also at finite temperatures is FFLO where the pairs form with a nonzero center of mass momentum. This is revealed clearly by a peak at nonzero momentum, $k_{\text {peak }}=\left|k_{\mathrm{F} 1}-k_{\mathrm{F} 2}\right|$, in the pair momentum distribution. The behavior of this peak is studied as a function of the temperature and also the polarization, $P$. We showed that increasing $P$ stabilizes FFLO up to higher temperatures and, in the confined case relevant to experiments on ultra-cold fermionic atoms, places this phase within reach.

Acknowledgments This work was supported by: an ARO Award W911NF0710576 with funds from the DARPA OLE Program; by the CNRS-UC Davis EPOCAL joint research grant; by NSF grant OISE0952300; by the France-Singapore Merlion program (PHC Egide, SpinCold 2.02.07 and FermiCold 2.01.09) and the CNRS PICS 4159 (France). Centre for Quantum Technologies is a Research Centre of Excellence funded by the Ministry of Education and the National Research Foundation of Singapore.
1 J. Bardeen, L.N. Cooper and J.R. Schrieffer, Phys. Rev. 108, 1175 (1957).

2 P. Fulde and A. Ferrell, Phys. Rev. 135, A550 (1964).

3 A. Larkin and Y.N. Ovchinnikov, Zh. Eksp. Teor. Fiz. 47, 1136 (1964) [Sov. Phys. JETP 20, 762 (1965)].

${ }^{4}$ G. Sarma, Phys. Chem. Solids 24, 1029 (1963); S. Takada and T. Izuyama, Prog. Theor. Phys. 41, 635 (1969).

5 V.L. Ginzburg and D.A. Kirzhnits, Soviet Phys. JETP 20, 1346 (1965); Nature 220, 148 (1968); R. Casalbuoni and G. Nardulli, Rev. Mod. Phys 76, 263 (2004).

6 M.W. Zwierlein it et al., Science 311, 492 (2006); M.W. Zwierlein et al., Nature 422, 54 (2006); Y. Shin et al., Phys. Rev. Lett. 97, 030401 (2006); Y. Shin, C.H. Schunck et al., Nature 451, 689 (2008).

7 G.B. Partridge et al., Science 311, 503 (2006); G.B. Partridge et al., Phys. Rev. Lett. 97, 190407 (2006).

8 Y. Liao et al. arXiv:0912.0092v1 [physics.atom-ph].

9 P. Castorina et al., Phys. Rev. A72, 025601 (2005); D.E. Sheehy and L. Radzihovsky, Phys. Rev. Lett. 96, 060401 (2006); J. Kinnunen, L.M. Jensen and P. Törmä, Phys. Rev. Lett. 96, 110403 (2006); K. Machida, T. Mizushima and M. Ichioka, Phys. Rev. Lett. 97, 120407
(2006); M.M. Parish et al., Nature Phys. 3, 124 (2007); H. Hu, X.-J. Liu and P.T. Drummond, Phys. Rev. Lett. 98, 070403 (2007); T. Koponen et al., New J. Phys. 8, 179 (2006); X.-J. Liu, H. Hu, P.D. Drummond, Phys. Rev. A76, 043605 (2007).

10 D.T. Son and M.A. Stephanov, Phys. Rev. A74, 013614 (2006).

11 G. Orso, Phys. Rev. Lett. 98, 070402 (2007).

12 G.G. Batrouni, M.H. Huntley, V.G. Rousseau and R.T. Scalettar, Phys. Rev. Lett. 100, 116405 (2008).

13 M. Casula, D.M. Ceperley, and E.J. Mueller, Phys. Rev. A78, 033607 (2008).

14 A. Feiguin and F. Heidrich-Meisner, Phys. Rev. B76, 220508(R) (2007); A. Lüscher, R.M. Noack, and A.M. Läuchli, Phys. Rev. A78, 013637 (2008); M. Rizzi et al., Phys. Rev. B77, 245105 (2008); M. Tezuka and M. Ueda, Phys. Rev. Lett. 100, 110403 (2008).

15 X. Liu, H. Hu, P.D. Drummond, Phys. Rev. A78, 023601 (2008); P. Kakashvili and C.J. Bolech, Phys. Rev. A79, 041603(R) (2009); T.K. Koponen et al., New J. Phys. 10 045014 (2008).

16 M. J. Wolak, V. G. Rousseau, C. Miniatura, B. Gremaud, 
R. T. Scalettar, G. G. Batrouni, arXiv:1004.4499, Phys. Rev. A82, 013614 (2010).

17 R. Blankenbecler, D.J. Scalapino, and R.L. Sugar, Phys. Rev. D24, 2278 (1981); S.R. White et al., Phys. Rev. B40, 506 (1989).
18 V.G. Rousseau, Phys. Rev. E 77, 056705(2008); V.G. Rousseau, Phys. Rev. E 78, 056707(2008).

19 G. G. Batrouni, M. J. Wolak, F. Hebert, V. G. Rousseau; Europhys. Lett. 8647006 (2009). 